\title{
Technical Note: REFIR-PAD level 1 data analysis and performance characterization
}

\author{
G. Bianchini and L. Palchetti \\ Istituto di Fisica Applicata "Nello Carrara" CNR-IFAC, Florence, Italy \\ Received: 1 October 2007 - Published in Atmos. Chem. Phys. Discuss.: 10 January 2008 \\ Revised: 24 June 2008 - Accepted: 24 June 2008 - Published: 21 July 2008
}

\begin{abstract}
The outgoing long-wave radiation from the Earth's atmosphere in the far infrared spectral region is mostly unexplored, while is well recognized that the water vapour contribution to greenhouse trapping is dominant in this region. The Radiation Explorer in the Far InfraRed (REFIR) study has proven the feasibility of a space-borne Fourier transform spectrometer able to perform the measurement in the $100-1100 \mathrm{~cm}^{-1}$ range with a resolution of $0.5 \mathrm{~cm}^{-1}$. Following this work a prototype of the spectrometer named REFIR-PAD (Prototype for Applications and Development) has been developed to observe the atmospheric radiance from both ground-based sites and from stratospheric balloon platforms. In this work we describe the REFIR-PAD level 1 data analysis procedure, that, starting from raw instrumental data produces the calibrated atmospheric spectral radiance. Performances of the procedure are also described.
\end{abstract}

\section{Introduction}

The study of the global climate change is one of the main objectives of Earth science for the coming decades and the collection of complete and accurate long-term data sets have become then necessary to answer to open scientific questions. The spectral measurement of atmospheric outgoing long wave radiance (OLR), and the far infrared (FIR) spectral region in particular, are one of the most interesting problems in this framework (Sinha and Harries, 1995).

The REFIR project, which was funded by the European Union (EU), addresses the feasibility of a Fourier transform spectrometer (FTS) suitable for a satellite mission aimed to the measurement of the Earth's spectral radiance extended to the FIR region, from 100 to $1100 \mathrm{~cm}^{-1}$ (REFIR final report, 2000). The availability of these measurements will improve

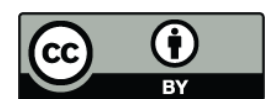

Correspondence to: G. Bianchini (gb@ifac.cnr.it) our knowledge of the interaction between radiation, water vapour and clouds in the upper troposphere (Rizzi et al., 2001). The FTS (Carli et al., 1999; Palchetti et al., 1999) is capable of resolving the OLR with a spectral resolution of $0.5 \mathrm{~cm}^{-1}$ and a signal-to-noise ratio greater than 100 in the $100-1100 \mathrm{~cm}^{-1}$ spectral range, with a $7 \mathrm{~s}$ acquisition time as required by the satellite platform.

A prototype of this FTS named REFIR-PAD (REFIR - Prototype for Applications and Development) (Bianchini et al., 2006a), has been developed at IFAC-CNR in order to test the instrument performances in near-space conditions. REFIR-PAD has been deployed on board a stratospheric balloon platform in the framework of the ELBC 2005 (Equatorial Large Balloons Campaign) measurement campaign, held during June 2005 in the North-East of Brazil (Bianchini et al., 2006b; Palchetti et al., 2006; Palchetti et al., 2008), and in various ground-based campaigns performed from highaltitude stations in Italy.

The instrument features dual input and output ports. One of the input ports observes a room temperature reference blackbody (RBB), while the other features a pointing mirror that can be switched between a hot and a cold calibration blackbodies (HBB and CBB respectively) and three different atmospheric views: nadir, limb and $30^{\circ}$ above horizontal. Each of the two output ports is provided with a Deuterated LAlanyne doped Triglycene Sulphate (DLATGS) pyroelectric detector with Winston cone collector.

The interferometer scanning mirror position is monitored by means of a reference laser interferometer using a temperature stabilized solid state semiconductor laser source at the wavelength of $780 \mathrm{~nm}$ (Bianchini et al., 2000). The maximum spectral resolution achievable by the system is $0.25 \mathrm{~cm}^{-1}$, corresponding to a maximum optical path difference of $2 \mathrm{~cm}$ (double-sided interferogram). A typical scan in the balloon flight configuration, at the operative resolution of $0.5 \mathrm{~cm}^{-1}$, is performed in about $30 \mathrm{~s}$.

This paper describes in detail the procedure used in order to perform the analysis of the raw instrumental data to

Published by Copernicus Publications on behalf of the European Geosciences Union. 


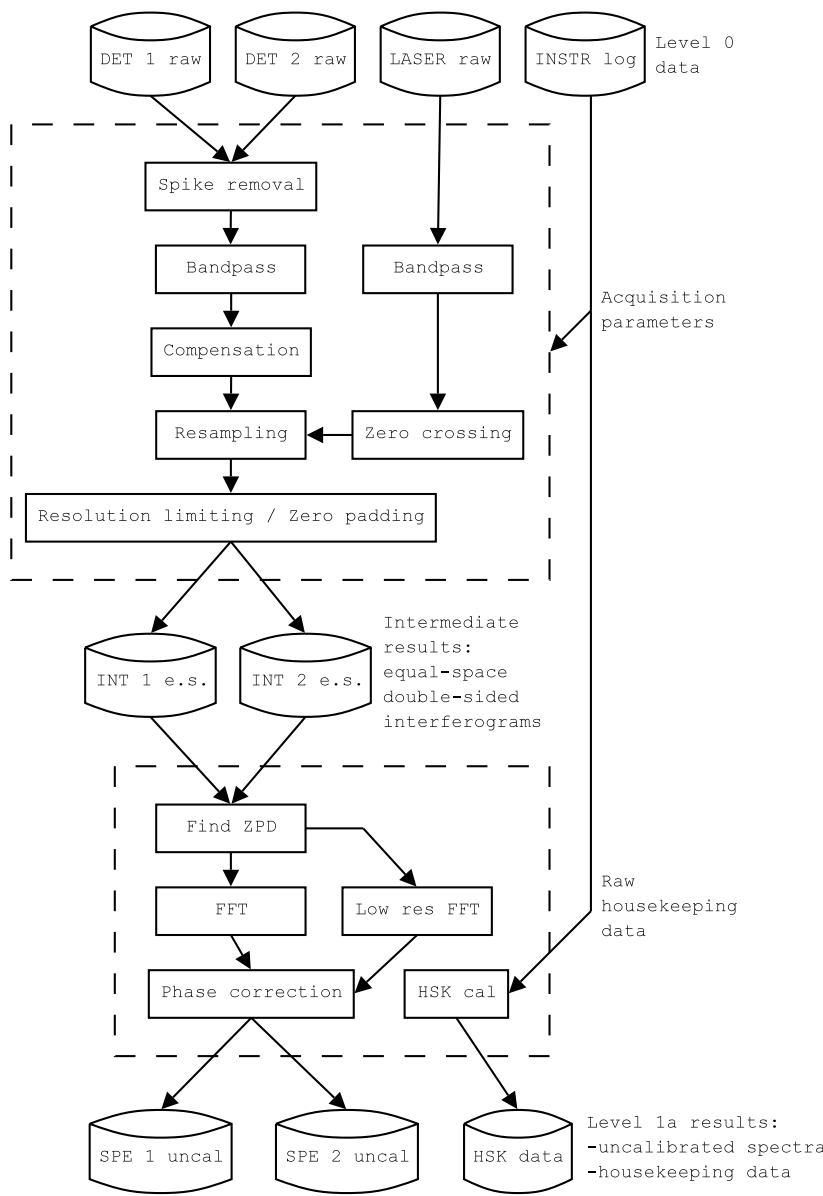

Fig. 1. Flowchart of the level 1a section of the REFIR-PAD data processing algorithm.

obtain the radiometrically and frequency calibrated atmospheric spectral radiance. In particular, in Sect. 2 we describe the Fourier transformation procedure providing the uncalibrated spectra from raw interferograms, while in Sect. 3 we discuss the calibration issues in order to obtain the final radiance products. In Sect. 4, we describe the characterization of the uncertainty on the measured radiance. Finally, in Sect. 5 we present the calibrated spectra and assess the instrument performances in the case of the stratospheric flight and the ground-based observations.

\section{Uncalibrated spectra}

The section of the REFIR-PAD data analysis workflow here identified as level 1a is peculiar of FTS instruments, and has the purpose of obtaining the uncalibrated spectra and the housekeeping data from the instrument raw data (level 0).

Since the interferogram sampling method used in REFIRPAD is at equal-time intervals, a first part of the level 1a data analysis is devoted to the filtering and resampling of the in-

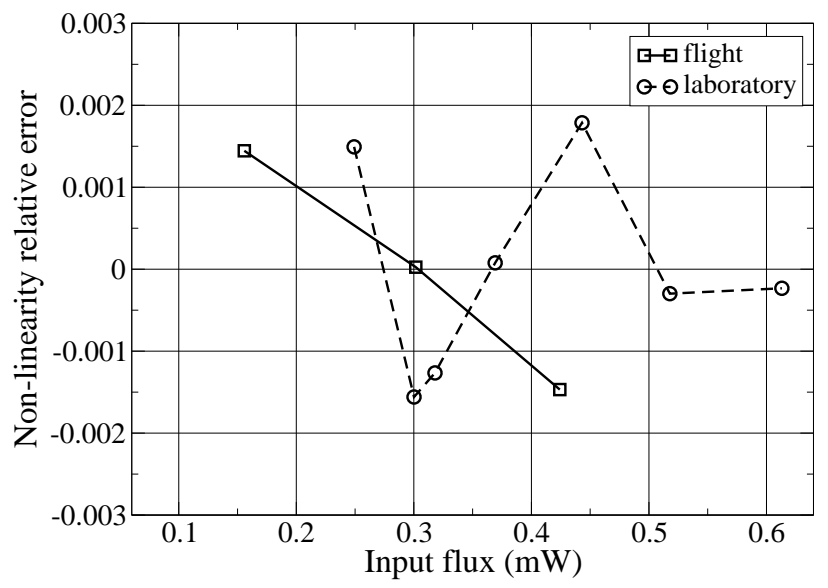

Fig. 2. Relative error due to detector non-linearity. The input flux variation is obtained by changing the temperature of the input blackbody sources, in laboratory under vacuum conditions (circles and dashed line) and during a stratospheric flight (squares and continuous line).

terferograms on equally spaced optical path difference intervals (Brault, 1996) following the interference fringes of the reference laser interferometer. The second part of the level 1a procedure actually performs the Fourier transform of the resampled interferograms and the phase correction. The calibration of the raw housekeeping data that carry ancillary information as reference blackbody temperatures, system temperatures and environmental parameters is performed in the third part of the level 1a procedure.

The flowchart of the level 1a procedure is shown in Fig. 1, with the different sections described above identified by blocks.

\subsection{Detector system characterization}

First of all a preliminary analysis has been performed on the detector and preamplifiers subsystem in order to verify that the achieved performances meet the requirements.

In the data analysis procedure described here, we assume that the detector system has a linear response through the full dynamic range experienced during REFIR-PAD measurements. The non-linearity was estimated by measuring the detector response to different values of the input radiation flux, obtained by varying the temperature of the source observed by the instrument. The response was then calculated by integrating the uncalibrated spectrum of the signal over the frequency range of interest for REFIR-PAD.

The non-linearity error was measured by calculating the relative difference from the linear response. In Fig. 2 the error is shown as a function of the input flux calculated as the total input power entering the instrument from both input channels, i.e. the radiation coming from the reference input blackbody at the constant temperature of the instrument, 
and from the measuring input port. This analysis was performed under vacuum in laboratory with the measuring input port looking at a blackbody source with temperatures in the range of 318-404 K (dashed-circle line). The figure also reports the results obtained during the stratospheric flight, where different fluxes correspond to the observation of the deep space, the reference sources CBB at $290 \mathrm{~K}$, and HBB at $350 \mathrm{~K}$ (continuous-square line).

The figure shows a non-linearity error less then $0.2 \%$, which produces a negligible effect on the calibrated radiance compared to radiometric uncertainties due to detector noise and the accuracy with which reference blackbody temperatures are known (see the results shown in Sect. 3).

Another requirement that has to be imposed to the detector subsystem is that the overall noise should be dominated by the intrinsic component due to the detector element, without any contribution from the preamplifier. In Fig. 3 noise spectra acquired with a network analyzer (Stanford Research SR-780) on the outputs of the detector subsystem are shown. Solid lines show the noise level due to the preamplifier only, while dashed and dotted lines show the response of the two acquisition channels with the two pyroelectric detectors connected to the preamplifiers.

As expected the electronics contribution is equal for the two channels and about $20 \mathrm{~dB}$ below detectors noise through the operating frequency range of the REFIR-PAD instrument (about $5-100 \mathrm{~Hz}$ ). The total noise spectrum is different for the two channels, and in agreement with the specified noise figures for each detector.

\subsection{Interferogram filtering and resampling}

With reference to Fig. 1, the interferograms from the two infrared detectors (DET 1 raw and DET 2 raw), and the reference interferometer (LASER raw) first are bandpass filtered to remove baseline effects and high frequency noise.

Since the pyroelectric detectors used for the infrared channels show sensitivity to acoustic and electronic transients, a procedure of identification and correction of transient phenomena is applied to the infrared signals. The system used is directly derived from the algorithm used to correct the cosmic ray transients detected by bolometric sensors (Lanfranchi et al., 1999), and makes use of wavelet transform for the identification of signal spikes (Bianchini et al., 2002).

In order to correctly perform the resampling, also a compensation of the acquisition system response is needed, since a time delay between reference laser and infrared signals leads to significant sampling errors. The laser detector and preamplifier can be assumed to have an ideal flat response, so the main source of delay is the phase of the infrared detection system response.

The pyroelectric detectors show a resonant behaviour with a strongly frequency dependent response that can be described with a low-pass and a high-pass filter whose cut-off

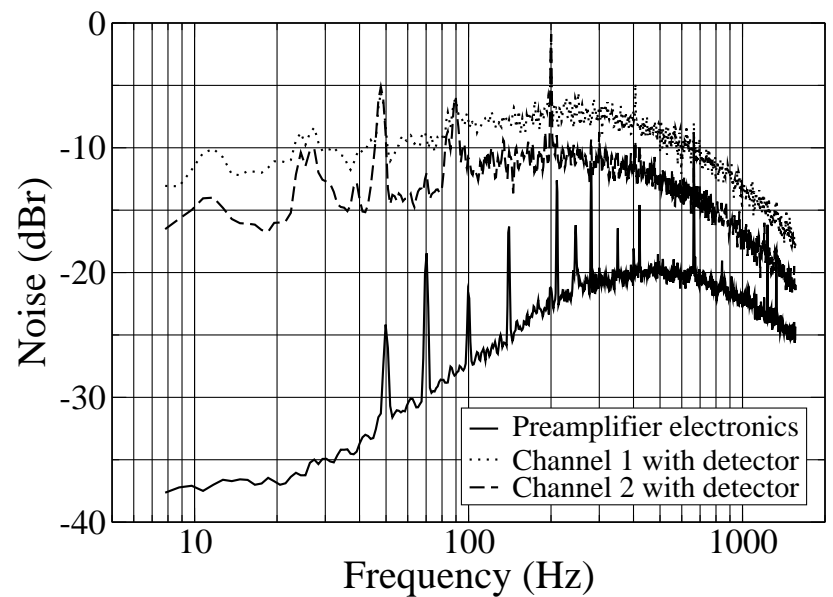

Fig. 3. Noise characteristics of the REFIR-PAD detector and preamplifier subsystem. Solid lines show the electronics contribution, while dashed and dotted lines represent the total noise (arbitrary units) of the two output channels.

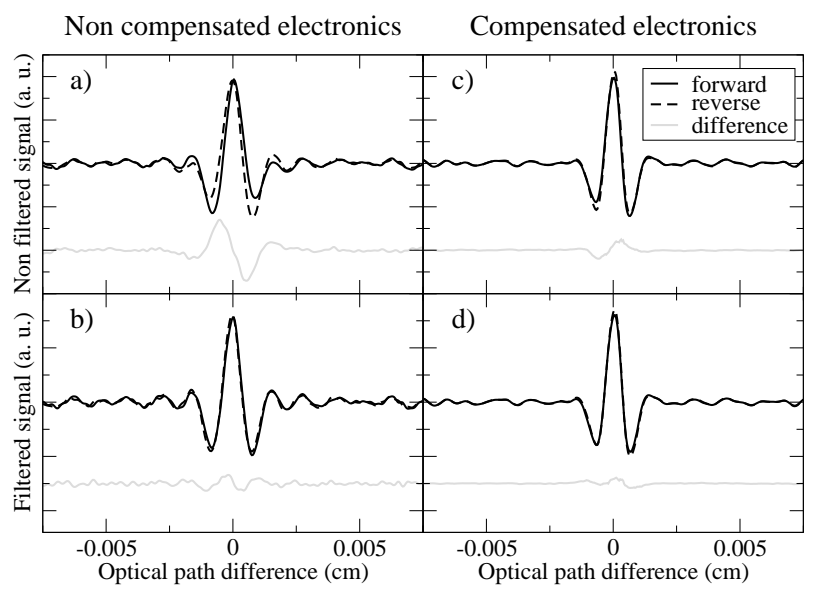

Fig. 4. Effect of electronic and software compensation of infrared detector response on interferogram symmetry. (a) non compensated electronics, no software correction. (b) non compensated electronics with software correction. (c) compensated electronics, no software correction. (d) compensated electronics with software correction. Differences between reverse and forward interferograms, offset for better readability, are also reported.

frequencies are "crossed", that is the low-pass cut-off is at lower frequencies than the high-pass.

The result is a peaked frequency response with a strongly non-linear phase that induces frequency-dependent time delays. The signal distortions due to the system response are identified by comparing two interferograms acquired as a function of the optical path difference (OPD) with opposite scanning directions.

As shown in Fig. 4a, inverting the scanning direction the distortions are reversed, since they are not OPD-dependent 


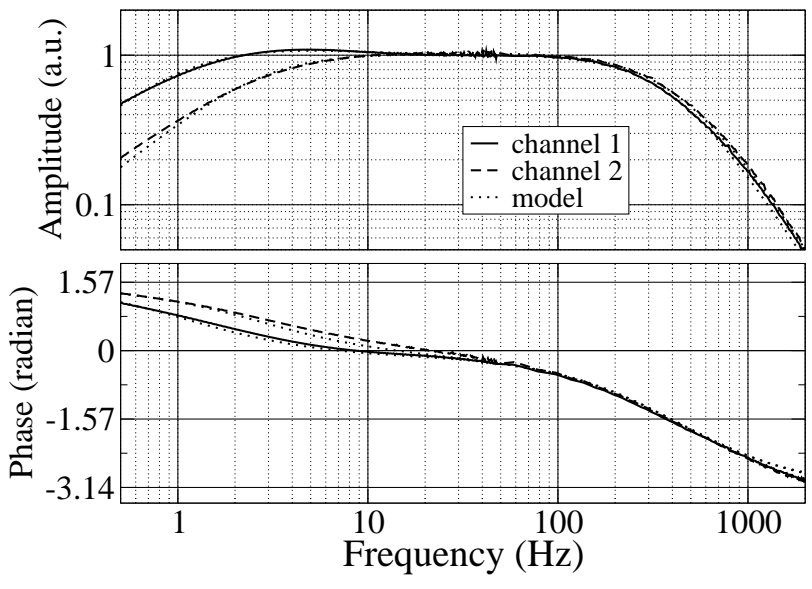

Fig. 5. Measured frequency response of the two channels of the REFIR-PAD infrared detection system (solid and dashed lines). The dotted lines show the modelled instrumental response used in the software compensation of electronic response.

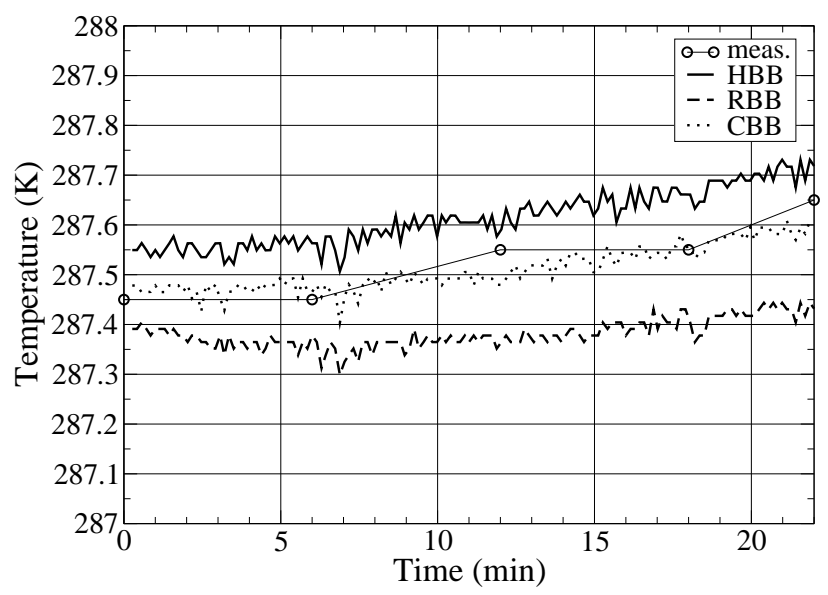

Fig. 6. Temperature measured by one of the PT100 sensor for each of the three blackbody reference sources, HBB (continuous line), RBB (dashed line), and CBB (dotted line). The circles show the average temperature values obtained by 3 external thermometers in thermal contact with the blackbodies.

but time-dependent. This time-dependent asymmetry can be corrected with an appropriate digital filter obtained from the measurement of the detector and preamplifier frequency response. After the application of the filter, the interferograms appear as shown in Fig. 4b.

It should be noted that there is also a residual asymmetry that is OPD-dependent. This effect has an optical origin, and will be corrected during the interferogram transformation through the phase correction procedure described in Sect. 2.3.

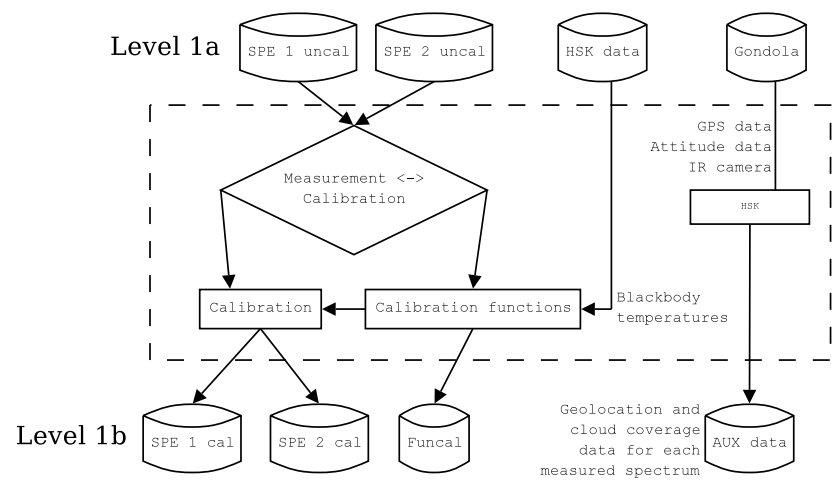

Fig. 7. Flowchart of the level $1 \mathrm{~b}$ section of the REFIR-PAD data processing algorithm.

A step further in the direction of compensating the detector response is through the preamplifier. The configuration used in the REFIR-PAD instrument includes a filtering network that flattens the resonant response of the detectors. The measured frequency response of the REFIR-PAD infrared detector system is shown in Fig. 5.

To implement the digital compensation, the detector plus preamplifier frequency response has been estimated through the response of the system to a step excitation given by a laser beam. Since pyroelectrics are thermal detectors, no difference in the response is expected using a visible source instead of an infrared one. In Fig. 5 a mathematical model of the response with the coefficients adjusted in order to fit the experimental response is shown. The model is used for the software compensation, even if, as expected, with the correct electronic filtering no big improvement is obtained through software compensation, as shown in Fig. $4 \mathrm{c}$ and d.

Finally, after the above described filtering, the infrared signal is resampled in the OPD domain on the zero crossing points of the reference interferometer signal. If a constant resolution is required, the interferograms are cut in order to obtain the desired resolution.

To avoid problems in the resampling process, laser signal is oversampled in the time domain. As an example, the sampling frequency used in the balloon flight configuration is $20 \mathrm{kHz}$, while the laser fringe frequency is about $1 \mathrm{kHz}$.

\subsection{Fourier transform and phase correction}

The next step involves performing the Fourier transform of the intermediate products, i.e. the equal-space resampled interferograms (INT 1 e.s. and INT 2 e.s. using the terminology of Fig. 1) in order to obtain the uncalibrated spectra (SPE 1 uncal and SPE 2 uncal).

The acquisition point of the interferogram that is nearest to ZPD is obtained through signal autocorrelation. Then a complex FFT is performed using that point as ZPD. The same interferogram with a greatly reduced resolution is used to 
obtain the frequency dependent phase, with which the full resolution spectrum is phase corrected (Mertz, 1967).

The advantage of this method is that only the low resolution information in the phase is retained, while the high resolution component (noise included) is rejected. In the alternative case of using a simple linear phase correction (Porter and Tanner, 1983), as produced by a simple ZPD point misplacement, a residual non linear phase error is still present. On the other side, by taking the modulus of the FFT, the spectral noise gives a bias error in the spectrum.

\subsection{Housekeeping data}

Auxiliary information required for diagnostic and instrument calibration is acquired through 20 housekeeping channels, sampled at a lower rate than the detectors (two times per acquisition, at the start and end of each interferogram) and stored as a raw 16-bit digital readout. Raw housekeepings are then converted into physical quantities through formulae and coefficients that are determined a-priori during laboratory tests.

Housekeeping signals can be divided in three categories, environmental monitoring, instrument diagnostics and blackbody temperature measurements. Environmental parameters as air temperature, relative humidity and pressure inside of the instrument are used to characterize possible systematic effects as absorption due to water vapour inside of the interferometric path. Instrumental parameters as the temperature of various instrument subsystems, the pressure inside the hard disk enclosure, the laser stabilization error signal and output power provide a diagnostic tool in case of instrument failure.

The housekeeping signals relative to the temperatures of the calibration sources are actually part of the level 1 data analysis workflow, and due to their key role, are monitored with accuracy and redundancy. For each source, three different PT100 sensors are used. The reading error was characterized in laboratory by comparing the measured values in each blackbody with the temperature measured by 3 external thermometers (Cole-Parmer Digi-Sense ${ }^{\circledR}$ model 8528-30 type $\mathrm{J}$ thermocouple thermometer) having $0.1 \mathrm{~K}$ accuracy, and posed in thermal contact with the blackbodies (Palchetti et al., 2008). The measurement was performed with the whole instrument kept in thermal equilibrium with the environment. Figure 6 shows, as an example, the comparison of the temperatures measured by a PT100 sensor for each source with the temperature measured by the 3 external thermometers, which give the same readout of about $287.6 \mathrm{~K}$. Similar results are obtained for the other PT100 sensors. The reading accuracy turned out to be within $0.3 \mathrm{~K}$.

\section{Calibration}

The level 1a data, consisting in uncalibrated spectra and calibrated housekeepings are then processed in order to calibrate spectra in terms of radiance, thus obtaining the level $1 \mathrm{~b}$ products (see Fig. 7).

Frequency calibration is performed using the reference laser (Bianchini et al., 2000). The reference source stability allows to use only a single measurement, performed using atmospheric line centers, in order to calculate laser frequency. The latter is then input as a parameter in the level 1a system and the resulting spectra are as a consequence frequency calibrated.

Radiometric calibration is however a more complex task and is obtained calculating the instrumental response using the on-board blackbody sources. If the instrument, the beam splitters and the RBB are at the same temperature, the beam splitter emission term can be considered negligible (Brasunas et al., 2002) and the instrument output is proportional to the difference of the two inputs with a frequency dependent complex proportionality factor here referred to as calibration function This condition is obtained if the product of instrumental emissivity and thermal anisotropies in the instrument are smaller than the required radiometric error (about $0.1 \mathrm{~K}$, as shown in Sect. 5), which is in general true for anisotropies of the order of few degrees.

Since in general the response of the two inputs can be different, depending on the optical layout characteristics, we consider two independent calibration functions $F 1(\sigma)$ and $F 2(\sigma)$. Complex spectra are used in order to take into account of some residual phase error that can affects the spectrum in narrow bands (Revercomb et al., 1988). Obviously, also the response of the outputs can be different, thus the following calibration procedure must be repeated for each output channel.

In these conditions for each output channel, the uncalibrated spectrum $S(\sigma)$, defined as the phase-corrected complex Fourier transform of the equal-space interferogram, is related to the calibrated radiance spectrum $L(\sigma)$ through the following complex relationship:

$$
S(\sigma)=F 1(\sigma) L(\sigma)-F 2(\sigma) B_{r}(\sigma)
$$

i.e.

$$
S(\sigma)=F 1(\sigma)\left[L(\sigma)-\frac{F 2(\sigma)}{F 1(\sigma)} B_{r}(\sigma)\right]
$$

where $B_{r}(\sigma)$ is the RBB radiance, equal to the theoretical Planck emission of a blackbody at the temperature of RBB.

$F 1(\sigma)$ and $F 2(\sigma)$ can be obtained from a two point radiometric calibration procedure performed by means of the measurement of the radiance of two calibration blackbodies, HBB (about $350 \mathrm{~K}$ ) and CBB (about $290 \mathrm{~K}$ ). 


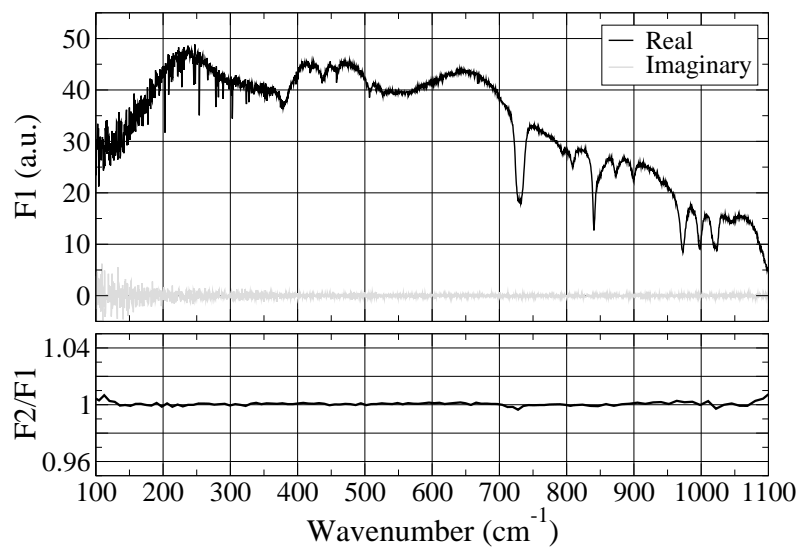

Fig. 8. The top panel shows the calibration function $F 1(\sigma)$ measured for the output channel 1 during a stratospheric balloon flight, real and imaginary parts (black and grey lines, respectively). In the bottom panel the real part of $F 2(\sigma) / F 1(\sigma)$ is shown.
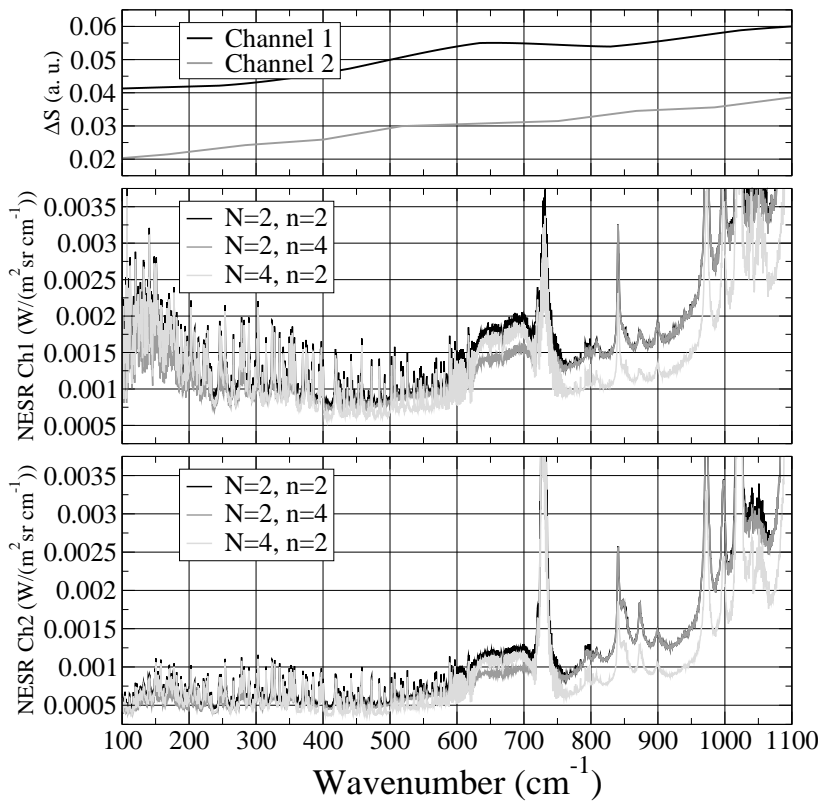

Fig. 9. Uncertainties on uncalibrated spectra (upper panel) obtained as standard deviation of multiple measurements, and NESR on calibrated spectra from error propagation in the case of nadir-looking geometry for stratospheric balloon observations (lower two panels) in the case of $32 \mathrm{~s}$ acquisitions.

If the temperature of RBB does not vary significantly between the two measurements $S_{h}$ and $S_{c}$ above described, we can obtain $F 1$ as:

$F 1(\sigma)=\frac{S_{h}(\sigma)-S_{c}(\sigma)}{B_{h}(\sigma)-B_{c}(\sigma)}$ where $B_{h}$ and $B_{c}$ are the theoretical Planck radiances respectively of $\mathrm{HBB}$ and $\mathrm{CBB}$.

The ratio $F 2(\sigma) / F 1(\sigma)$ depends on the response of the two independent inputs that can be slightly different due to small optical asymmetry in the interferometer inputs (mainly beam splitters asymmetry and different number of reflections). It can be characterized in laboratory and used in the on-board calibration as a known frequency-dependent factor.

Figure 8 shows an example of the spectrum of $F 1(\sigma)$ measured for the output channel 1 during the Brazilian stratospheric flight and $F 2(\sigma) / F 1(\sigma)$ as characterized for the used instrument configuration.

The real part of $F 1$ is modulated by the absorption in the optical path inside of the instrument, which is due to the beam splitter substrate (PET) and the infrared-absorbing air components (mainly water vapour). At the pressure conditions of the stratospheric flight, the contribution of water vapour to the instrumental absorption is very low, and the imaginary part is very small with respect to the real part. This small effect is, however, taken into consideration with the complex calibration.

Finally the calibrated radiance $L(\sigma)$ is calculated from the uncalibrated spectrum $S(\sigma)$ and the theoretical expression of $B_{r}(\sigma)$ by means of the following equation:

$L(\sigma)=\Re\left\{\frac{S(\sigma)}{F 1(\sigma)}+\frac{F 2(\sigma)}{F 1(\sigma)} B_{r}(\sigma)\right\}$

It should be noted that in general all the quantities used in the calibration procedure, are complex, and only in the last expression Eq. (4), the real part of the result is taken, obtaining, as needed, the measured spectrum as a real quantity.

\section{Radiometric uncertainty}

The absolute radiometric uncertainty on the calibrated spectra depends on the measurement precision and the accuracy with which the calibration procedure is performed. The measurement precision is calculated in term of the noise equivalent spectral radiance (NESR), which is dominated by the detector noise (random error component) and produces independent fluctuations for each spectral element, whereas the calibration accuracy is dominated by the measurement accuracy of the reference blackbody temperatures (systematic error component) since, as shown in Palchetti et al. (2008), their emissivity can be taken equal to 1 . In this latter case, the radiometric error in the different spectral elements is correlated.

\subsection{Noise equivalent spectral radiance}

The NESR was calculated from the $1 \sigma$ uncertainty on the uncalibrated spectrum as a function of the frequency. The uncalibrated error can be measured provided that we are able 

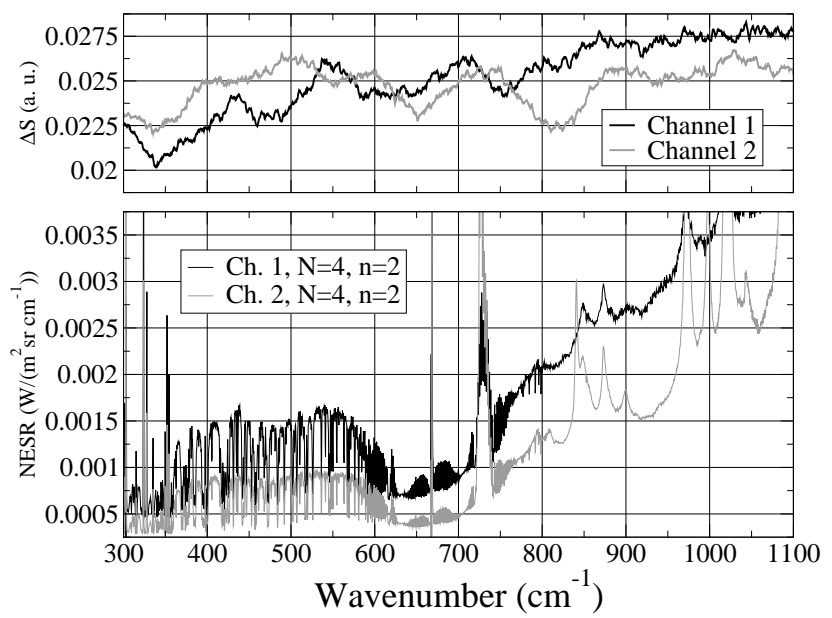

Fig. 10. Uncertainties on uncalibrated spectra (upper panel) obtained as standard deviation of multiple measurements, and NESR on calibrated spectra from error propagation in the case of zenithlooking geometry for ground-based observations (bottom panel) in the case of $64 \mathrm{~s}$ acquisitions.

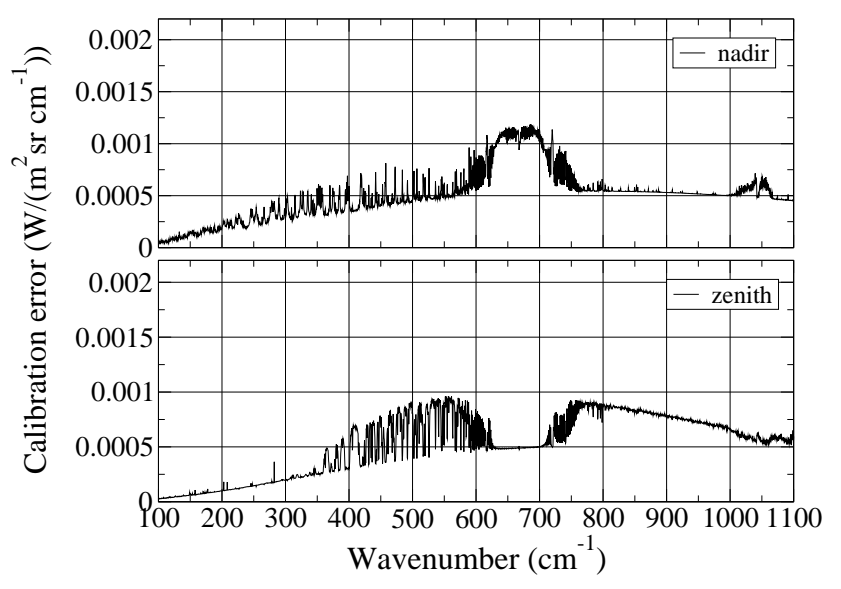

Fig. 11. Systematic calibration error due to a $0.3 \mathrm{~K}$ temperature uncertainty on the reference blackbody. In the top panel the error is shown in the case of nadir-looking geometry for stratospheric balloon observations. In the bottom panel the error is calculated in the case of zenith-looking geometry for ground-based observations..

to maintain enough stability in the reference and the measurement source while acquiring multiple spectra. With the instrument in thermal equilibrium, and using HBB as source, the requirements are achieved and the uncertainty for each spectral element in an uncalibrated spectrum is simply obtained from the standard deviation of multiple measurements. The result, smoothed in order to eliminate high frequency dependence, is shown in Fig. 9 (upper panel).

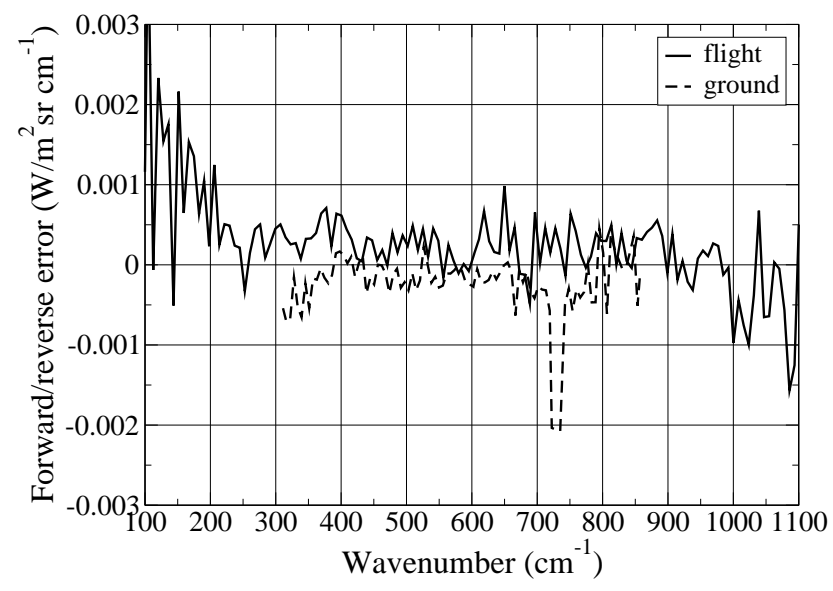

Fig. 12. Calibration error due to differences between scan directions measured in the case of the stratospheric flight (continuous line) and the ground-based observations (dashed line).

The value of NESR on each output channel can be finally obtained from the uncalibrated error through error propagation, using Eq. (4) with the approximation $F 2 / F 1 \simeq 1$. If we consider a general case in which the measured spectrum $S$ is the average of $N$ acquisitions, and the calibration measurements $S_{h}$ and $S_{c}$ come from an average of $n$ single spectra, respectively (i.e. $n$ is the number of calibration sequences), we obtain that:

$\mathrm{NESR}=\sqrt{\frac{1}{N}+\frac{2}{n}\left(\frac{S}{S_{h}-S_{c}}\right)^{2}} \frac{\Delta S}{F 1}$,

where $S, S_{h}, S_{c}$ are the averages respectively of the measured radiance, the $\mathrm{HBB}$ radiance and the $\mathrm{CBB}$ radiance and $\Delta S$ is the $1 \sigma$ uncertainty on the uncalibrated measurement. The results, calculated for different values of $N$ and $n$ and for a measured radiance equal to the nadir view as observed from stratospheric altitude are shown in Fig. 9 (lower two panels).

It should be noted that varying $N$ and $n$ different parts of the uncertainty spectrum are affected: in fact, the term $S /\left(S_{h}-S_{c}\right)$ contains a spectral dependence, and where it is small, i.e. the measured radiance is near the reference blackbody emission, the number of measurements $N$ predominates. In the other case, a reduction of uncertainty is better obtained through a greater number $n$ of calibrations.

We see that $n$ predominates in the $\mathrm{CO}_{2}$ region between 600 and $750 \mathrm{~cm}^{-1}$, while in the remaining regions of the REFIRPAD operating range, the effect of $N$ is bigger. The condition $N=4, n=2$ gives the best compromise with the minimum spectral variability on the calibrated uncertainty.

The uncertainty estimation procedure has been applied also to the zenith-looking geometry for ground-based observations and the results are shown in Fig. 10. In this case it should be noted that the uncalibrated error is lower due to 


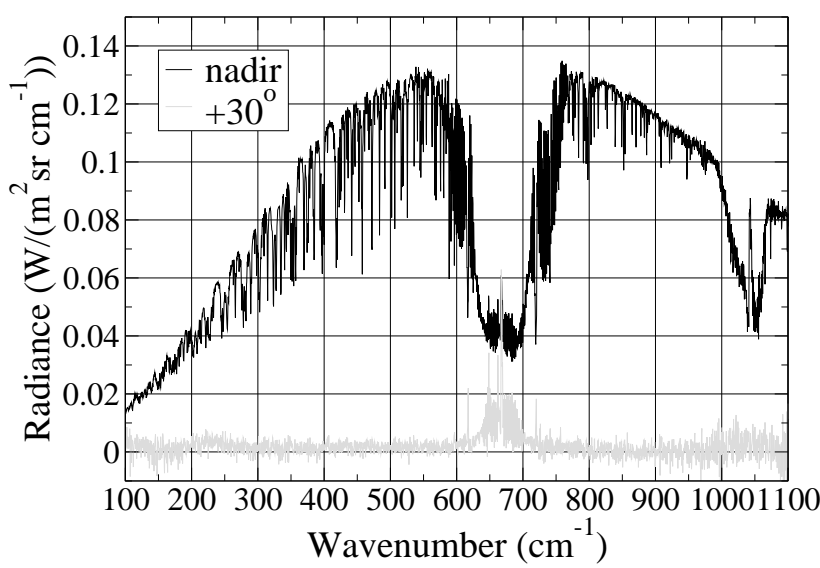

Fig. 13. Calibrated spectra in the case of the stratospheric flight. Spectra are obtained from channel 1 by averaging 4 acquisition sequences in clear sky conditions, 40 spectra for the nadir observing direction (black line) and 4 spectra for the $30^{\circ}$ above horizontal direction (gray line).

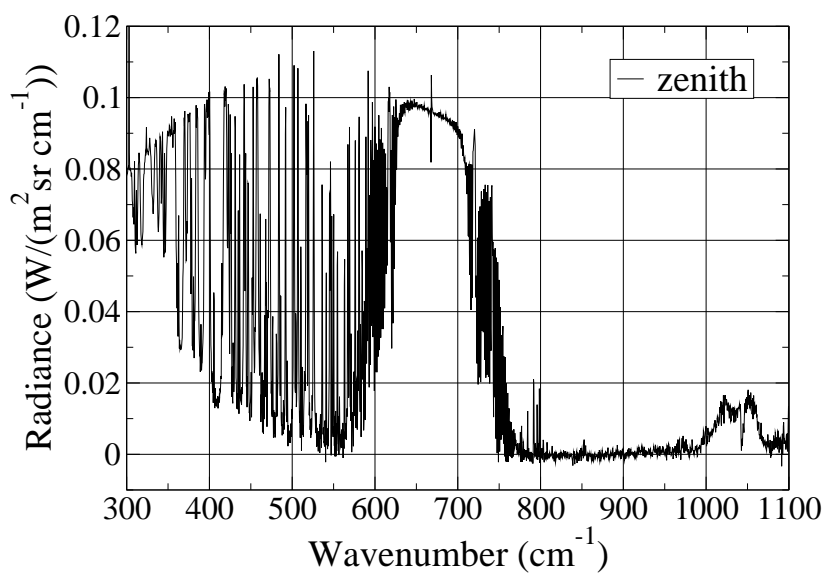

Fig. 14. Calibrated spectrum in the case of ground-based observations. Spectrum is obtained from the weighted average of both output channels of a single acquisition sequence of 4 zenith observations in clear sky conditions.

the higher integration time that was allowed by slower atmospheric variability observed by the instrument from a fixed position.

\subsection{Systematic calibration error}

The systematic calibration error was obtained through the error propagation of the temperature $1 \sigma$ error measured on the reference blackbodies. If we consider the case in which the temperature error of each reference blackbody is independent and that the corresponding uncertainty on the theoretical Planck emission is given by $\Delta B_{h}, \Delta B_{c}$, and $\Delta B_{r}$ for $\mathrm{HBB}$,
$\mathrm{CBB}$, and $\mathrm{RBB}$ respectively, we obtain the calibration error $\Delta L$ from equation:

$$
\Delta L=\sqrt{\Delta B_{r}^{2}+\left(\frac{S}{S_{h}-S_{c}}\right)^{2}\left(\Delta B_{h}^{2}+\Delta B_{c}^{2}\right)} .
$$

Figure 6 shows that a value of $0.3 \mathrm{~K}$ can be considered as a good conservative estimation of the temperature $1 \sigma$ error for the calculation of the blackbody emission uncertainty for the different heating conditions. The resulting $\Delta L$, calculated from Eq. (6), is shown in Fig. 11 as a function of wavenumber for the two different observation geometries, the nadir view (top panel) and the zenith view (bottom panel).

\subsection{Dependence of calibration on scan direction}

The response of the system depends slightly on the sweep direction since the phase error caused by detector response and possible misalignments can have different effects in the two directions and these effect cannot be completely corrected by numerical procedure. This can be taken into account by calibrating the forward and reverse sweep directions with separated calibration functions measured with the same sweep direction. The residual error due to this effect has been evaluated by measuring the difference in calibrated spectra between the forward and reverse sweeps. The results, in the case of both stratospheric flight (continuous line) and ground-based observations (dashed line), are shown in Fig. 12 for one output channel. The other channel has the same behaviour.

The resulting errors are below the NESR level of the measurement over the whole useful bandwidth (see Figs. 15 and 16), and no important systematic effect is introduced even if only one single sweep direction is used for the analysis.

\section{Calibrated spectra}

In Fig. 13 two typical spectra acquired during a stratospheric balloon flight, corresponding to the nadir and the $30^{\circ}$ above horizontal lines of sight are shown. Each spectrum is obtained from the average of 4 acquisition sequences each one including 10 nadir observations, one space view and 2 calibrations. Each observation has a $32 \mathrm{~s}$ duration, the total acquisition time is $1 \mathrm{~h}$, and the atmospheric conditions during acquisition were clear sky. Only one output channel is used for the analysis of the stratospheric flight since the second channel showed a degradation of performances due to an occasional thermal instability.

The large field of view of the instrument $(133 \mathrm{mrad})$ does not require a high accuracy in the pointing of this instrument. In fact, during the balloon flight the gondola oscillations were well inside the field of view, and an active line of sight stabilization was not required. 


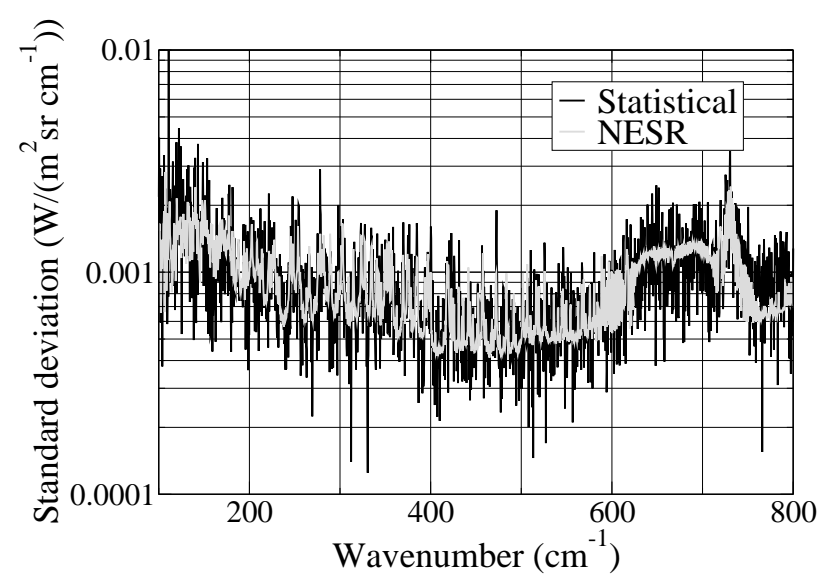

Fig. 15. Comparison in the case of the stratospheric flight between the estimated NESR and the standard deviation of 7 measurements, each of them obtained from a single sequence composed of 10 nadir observations and 2 calibrations.

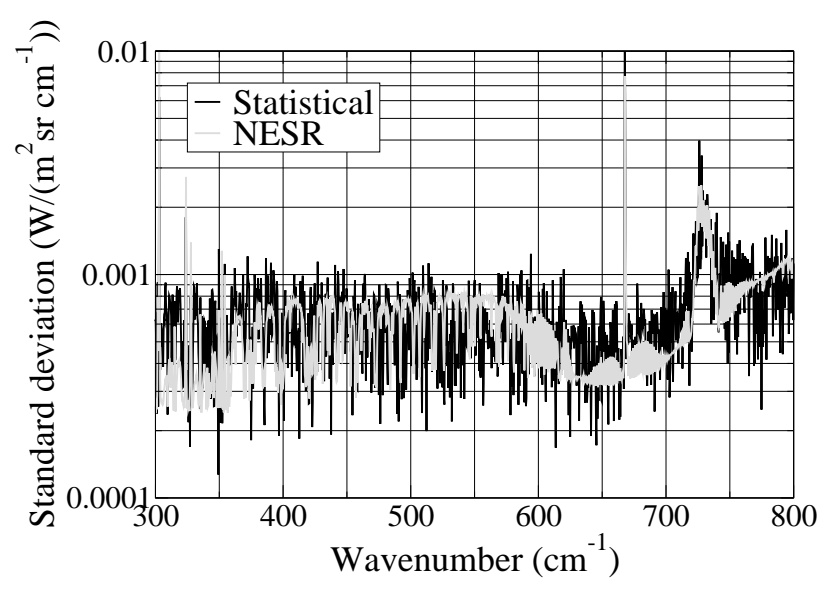

Fig. 16. Comparison in the case of ground-based observations between the estimated NESR and the standard deviation of 6 measurements, each of them obtained from the weighted average of both output channels of a single sequence composed of 4 zenith observations and 2 calibrations.

A self validation of the calibration procedure is provided from the space view, which, apart from the residual carbon dioxide band around $670 \mathrm{~cm}^{-1}$ and few water vapour lines, shows an atmospheric emission near to zero, as expected. The difference from zero of the space view radiance is converted in brightness temperature error with respect to a $280 \mathrm{~K}$ blackbody in order to evaluate the absolute calibration error. The result shows that the average calibration error during flight is about $0.1 \mathrm{~K}$ (Palchetti et al., 2006).

In Fig. 14 a spectrum acquired in the case of zenithlooking geometry from ground-based high-altitude location is shown. The spectrum is obtained from the weighted aver-

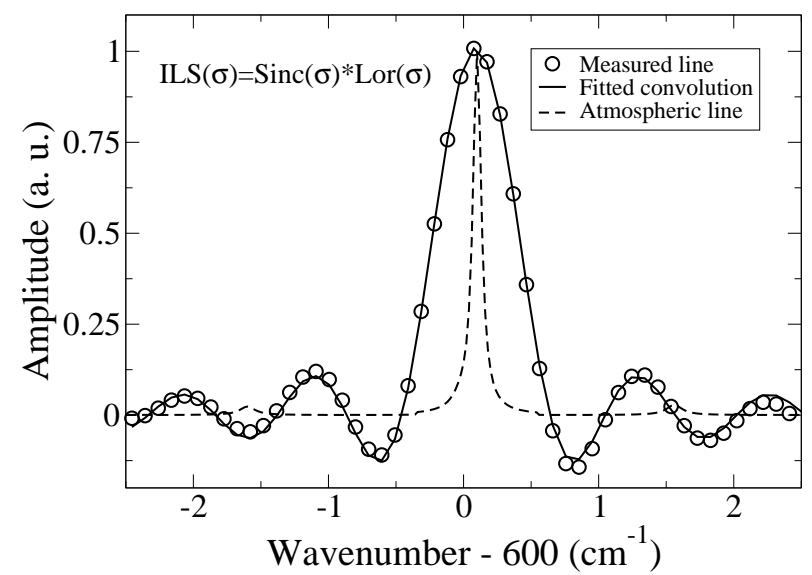

Fig. 17. Instrumental line shape as obtained from laboratory measurements (circles). The atmospheric line shape contribution (dashed line) and the fit with the theoretical function ILS $(\sigma)$ (continuous line) are also shown.

age of both output channels for a single acquisition sequence including 4 zenith observations, and 2 calibrations. Each observation has a duration of $64 \mathrm{~s}$, the total acquisition time is about $10 \mathrm{~min}$, and also in this case the atmospheric conditions during acquisition were clear sky.

The estimated uncertainty in the measured spectra can also be validated, through the comparison between the estimated NESR obtained in Sect. 4 and the statistical standard deviation of the measured spectra in presence of a stable atmospheric scene.

In Fig. 15 the standard deviation calculated from 7 measurements each obtained by averaging the spectra of a single acquisition sequence in the case of the stratospheric flight is shown. In the same graph the NESR corresponding to setting the values of $N=10$ and $n=2$ in Eq. 5 is plotted. The agreement is remarkable, and the only spectral region where the statistical variance of measurements is higher than the NESR is the atmospheric transparency window, where ground temperature variations and the possible presence of residual low clouds induce scene variability.

In Fig. 16 the same procedure is applied to ground-based measurements performed in stable atmospheric conditions. In this case, according to the measurement conditions the NESR is calculated for $N=4, n=2$, while the standard deviation is calculated from 6 measurements each obtained by averaging the spectra of a single sequence composed of 4 zenith observations and 2 calibrations. Also in this case the window region is not shown as it is influenced from residual atmospheric variability due to thin clouds.

A further product of level 1 data analysis is the instrumental line shape (ILS), that is fundamental for the following phases of data processing, the level 2 data analysis for the retrieval of atmospheric parameters. The REFIR-PAD ILS has 
been measured through laboratory spectra, using an isolated water vapour line with a spectral width much smaller than instrumental resolution. The REFIR-PAD spectrum of the selected line is shown in Fig. 17. In the same figure a fit of the theoretical line shape ILS $(\sigma)$ obtained from the convolution of a Sinc function and the atmospheric line shape $\operatorname{Lor}(\sigma)$ is shown. The good agreement obtained with this analysis shows that the instrumental self-apodization effect due to beam aperture is negligible, since the ILS is a pure Sinc function corresponding to the nominal resolution of $0.5 \mathrm{~cm}^{-1}$.

\section{Conclusions}

The level 1 data processing workflow for the REFIR-PAD instrument has been completed and validated, the results show that instrumental performances are well up to the scientific requirements that were posed from the REFIR preliminary study.

The REFIR-PAD spectroradiometer is capable to provide atmospheric emission spectra in the nadir, limb and space view lines of sight with an absolute radiometric calibration error of the order of $0.1 \mathrm{~K}$.

Laboratory measurement and instrument characterization obtained from in-flight data show a remarkable agreement, providing self-validation of both the instrumental set-up and the level 1 data analysis system.

Acknowledgements. The authors wish to thank the CNES balloon team (led by P. Chadoutaud) and the CNES nacelles pointèes team (led by J. Evrard), the LPMAA gondola team for having hosted REFIR-PAD, INPE for logistics in Brazil, and the PRIN project \#2005025202 led by C. Serio for the ground-based measurements in Italy.

Edited by: U. Pöschl

\section{References}

Bianchini, G., Lanfranchi, M., and Cortesi, U.: Flight qualification of a diode laser for path difference determination of a high-resolution Fourier transform spectrometer, Appl. Optics, 39, 962-965, 2000.

Bianchini, G., Cortesi, U., Palchetti, L., and Pascale, E.: Cosmic ray spikes localization and correction in FT spectrometer data, Infrared Phys. Techn., 43, 33-38, 2002.

Bianchini, G., Palchetti, L., and Carli, B.: A wide-band nadirsounding spectroradiometer for the characterization of the Earth's outgoing long-wave radiation, Sensors, in: Systems and Next-generation Satellites XII, edited by: Meynart, R., Neeck, S. P., and Shimoda, H., Proceedings of the SPIE, 6361, 63610A, 2006.

Bianchini, G., Palchetti, L., Belotti, C., Del Bianco, S., and Cortesi, U.: Wide-band spectrally resolved measurement of the Earth's up-welling radiation with the REFIR-PAD spectroradiometer, Remote Sensing of Clouds and the Atmosphere XI, edited by:
Slusser, J. R., Schäfer, K., and Comerón, A., Proceedings of the SPIE, 6362, 63622K, 2006.

Brasunas, J. C.: Phase anomalies in Fourier-transform spectrometers: an absorbing beam splitter is neither sufficient nor necessary, Appl. Optics, 41, 2481-2487, 2002.

Brault, J. W.: New approach to high-precision Fourier transform spectrometer design, Appl. Optics, 35, 2891-2896, 1996.

Carli, B., Barbis, A., Harries, J. E., and Palchetti, L.: Design of an efficient broadband far-infrared Fourier-transform spectrometer, Appl. Optics, 38, 3945-3950, 1999.

Lanfranchi, M., Carli, B., Gignoli, A., Lee, C., and Ridolfi, M.: Cosmic-ray flux detected by an IR bolometer operated on board of a stratospheric aircraft, Infrared Phys. Techn., 40, 379-386, 1999.

Mertz, L.: Auxiliary computations for Fourier spectrometry, Infrared Phys. Techn., 7, 17-23, 1967.

Palchetti, L., Barbis, A., Harries, J. E., and Lastrucci, D.: Design and mathematical modelling of the space-borne far-infrared Fourier transform spectrometer for REFIR experiment, Infrared Phys. Techn., 40, 367-377, 1999.

Palchetti, L., Belotti, C., Bianchini, G., Castagnoli, F., Carli, B., Cortesi, U., Pellegrini, M., Camy-Peyret, C., Jeseck, P., and Té, Y.: Technical note: First spectral measurement of the Earth's upwelling emission using an uncooled wideband Fourier transform spectrometer, Atmos. Chem. Phys., 6, 5025-5030, 2006, http://www.atmos-chem-phys.net/6/5025/2006/.

Palchetti, L., Bianchini, G., Carli, B., Cortesi, U., and Del Bianco, S.: Measurement of the water vapour vertical profile and of the Earth's outgoing far infrared flux, Atmos. Chem. Phys., 8, 28852894, 2008, http://www.atmos-chem-phys.net/8/2885/2008/.

Palchetti, L., Bianchini, G., and Castagnoli, F.: Design and characterisation of black-body sources for infrared wide-band Fourier transform spectroscopy, Infrared Phys. Techn., 51, 207-215, 2008.

Porter C. D. and Tanner, D. B.: Correction of phase errors in Fourier spectroscopy, Int. J. Infrared Milli., 4, 273-298, 1983.

REFIR - Radiation Explorer in the Far InfraRed: Final Report ENV4-CT6-0344, European Commission, Brussels, Belgium, 2000.

Revercomb, H. E., Buijs, H., Howell, H. B., LaPorte, D. D., Smith, W. L., and Sromovsky, L. A.: Radiometric calibration of IR Fourier transform spectrometers: solution to a problem with the High-Resolution Interferometer Sounder, Appl. Optics, 27, 3210-3218, 1988.

Rizzi, R., Carli, B., Harries, J. E., Leotin, J., Serio, C., Sutera, A., Bizzarri, B., Bonsignori, R., and Peskett, S.: Mission objectives and instrument requirements for the (REFIR) Radiation Explorer in the Far-InfraRed mission: an outline after the end of phase B0, in: Current Problems in Atmospheric Radiation, edited by: Smith, W. L. and Timofeyev, Y. M., Proc. of Int. Radiation Symp. (IRS) 2000, 567-570, 2001.

Sinha, A. and Harries, J. E.: Water vapour and greenhouse trapping: The role of far infrared absorption, Geophys. Res. Lett., 22, 2147-2150, 1995. 